九州大学学術情報リポジトリ

Kyushu University Institutional Repository

\title{
Kinematic Analysis of the ABB IRB 1520 Industrial Robot Using RoboAnalyzer Software
}

Shai lendra Singh Chauhan

G. L. Bajaj Institute of Technology \& Management

Avadhesh Kumar Khare

Quantum University

https://doi.org/10.5109/4150470

出版情報 : Evergreen. 7 (4)，pp.510-518，2020-12. 九州大学グリーンテクノロジー研究教育センター バージョン：

権利関係 : 


\title{
Kinematic Analysis of the ABB IRB 1520 Industrial Robot Using RoboAnalyzer Software
}

\author{
Shailendra Singh Chauhan ${ }^{1}$, Avadhesh Kumar Khare ${ }^{2}$ \\ ${ }^{1}$ G. L. Bajaj Institute of Technology \& Management, Greater Noida, Gautam Buddha Nagar, \\ U.P. 201310, India, shailendra.chauhan@glbitm.ac.in, sschauhan3456@gmail.com \\ ²uantum University, Roorkee, India, advisor@quantumuniversity@edu.in, khareavadhesh@gmail.com
}

*Author to whom correspondence should be addressed:

E-mail: sschauhan3456@gmail.com

(Received September 4, 2020; Revised December 8, 2020; accepted December 16, 2020).

\begin{abstract}
Robots are extensively used in various industries in manufacturing sector for different operations consistently. Some robots are having simulation software for offline programming, but few other robots do not provide this facility for the purpose of analysis. Various parameters and characteristics of robot are important to understand for obtaining its better performance. The inverse and forward kinematics is important to understand the dynamics of the robot required for the design and control. Therefore, RoboAnalyzer software is used to visualize the robot kinematics in simulated environment prior to executing the actual motion of the robot. In the present study a mathematical model for forward kinematics of ABB IRB- 1520 industrial robot is developed and the results are verified by using RoboAnalyzer. The forward kinematics equation is written using Denavit-Hartenberg analysis (D-H). Both the geometrical and the analytical methods are used to solve the problem of inverse kinematics. It calculates the required joint angles $\left(\theta_{1}\right.$ to $\left.\theta_{6}\right)$. The proposed method in this paper is generic in nature, and can be applied to carry out kinematic analyses of any robot manipulator.
\end{abstract}

Keywords: D-H parameters, Forward Kinematics, Inverse Kinematics, RoboAnalyzer, ABB IRB1520 industrial robot

\section{Introduction}

During the last few years the demand and the application of industrial robots have seen a drastic uplift. In the manufacturing operations the robotics technology has reduced the production cost, material wastage and boost up the productivity by reducing the cycle time and giving better quality. They are being used for various activities like painting, assembly, welding, pick and place and are being used in a very wide range of applications ${ }^{1)}$. The basic reasons for such a high demand of these industrial robots are their accuracy and their work capacity $^{2)}$. Robots have a wide range of applications in the manufacturing sectors due to its flexibility and programmability. There are dozens of benefits that can be extracted with the help of an industrial robot. They can give results with high precision and minimal errors ${ }^{3)}$. The basic use of industrial robots involves a wide range of application out of which robot manipulator is the one that is used extensively in the production and assembly plants mainly. In medical applications such as distance surgery robots manipulators are mostly used because of its precision motion. These robot manipulators are generally humanoids or anthropomorphic yet there are many of them that can be seen and considered as electromechanical from the structural point of view. Robot manipulators move across a predefined path that is created with the help of end effector and orientation of points. In a Robot manipulator generally the human arm, brain and Heart are being copied, also known as 3H's. Robot manipulators move across a predefined path which is created with the help of teach pendant and orientation of points. This path is then traced by the end-effector. The working region or the Cartesian space is a function of time. These industrial robots are a rigid bodies system, called links that are connected through the kinematic joints. These joints can be of many types that allow some kind of relative motion between the two links. These links, connected with the help of kinematic joints, are termed as a kinematic chain. These kinematic chains can be open, closed or branched. In the open kinematic chains the position and orientation of the output point is determined by the series of transformations of the joints connecting the forward path from input link to output point ${ }^{4,5)}$. Thus, the Forward Kinematics (FKin) of open 
kinematic chains provides a unique solution, and the study is called Kinematic Analysis ${ }^{6)}$. On the contrary, if the position and orientation of the output point is prescribed, then determining the transformations of each connecting joint is an Inverse Kinematics (IKin) problem, and falls in the category of Kinematic Synthesis. Unlike Kinematic Analysis, Kinematic Synthesis results into multiple solutions to be compared based on some other criteria $^{7)}$. For performing smooth operations, it is required to compute all the important points in a Cartesian co-ordinate. Inverse kinematics is referred to the conversion of trajectory locations from Cartesian coordinate to joint coordinates. In simple words Inverse kinematics is referred to calculating the joint angles required to get the required position and end effector (EE) orientation ${ }^{8)}$. The precision can only be achieved if and only if all the factors and the processes are taken into consideration. The forward kinematics is a method that is related to the develop relationship between robot joint angles and the final location and orientation of the EE. The position of EE is finally determined on the basis of points and locations along with the joint angles ${ }^{9)}$.

Manipulator Kinematics is the analysis of motion i.e. kinematic parameters without considering the forces/torques that causes the motion ${ }^{10)}$. If a robot is to perform a specific task, the location and end-effector orientation with reference to the base should be established first. The configurations determined by the six Cartesian variables that are controlled by the robot's joint motions, it is necessary to find the relations between the two sets. Therefore robot kinematics is divided in to two fundamental tasks namely forward kinematics (FKin) and inverse kinematics (IKin) ${ }^{10)}$. FKin is referred to going from joint space to world space. Whereas, IKin refers to going from world space to joint space $^{11)}$. The position and orientation of the tool frame with respect to the global reference frame is determined by a homogeneous transformation matrix (TM) which has to be developed. Same TM is used for FKin as well as IKin to solve the problem. A 6R manipulator IKin was first described by Pieper ${ }^{11)}$. He introduced iterative numerical techniques and solved a problem of upper limits for the total number of solution using an elimination theory. In this work the ABB IRB 1520 Industrial Robot is used for FKin and IKin analysis and validates its results with the use of software Robo Analyzer developed at Robotics and Mechatronics lab IIT Delhi. Animation analysis and end effector trajectories of robot are presented in the form of graph plots.

\section{Experimental Setup}

The ABB IRB 1520 is an articulated welding Robot having six axis (6DOF) industrial manipulators as shown in figure 1(a). The robot is fixed with its base and the other linkages and end effector is free to move in the space. The robot manipulator dimensions and centre to centre distances between linkages are presented in the figure 1(b). First three axes of the robot i.e. A1, A2 and A3 in figure 2 are major axes because these axes are contributing to the positioning, whereas other three axes i.e, A4, A5 and A6 are called minor axes which are used for the articulating the end effector to the target point where the minor axes contribute towards orientation of the end-effector. This robot has a compact process where the upper arm has designed for integrated dressing packages for arc welding with excellent accuracy and it has a work handling capacity of $4 \mathrm{Kg}$ with an armload of $10 \mathrm{Kg}$. This type of robot is mainly used for the arc welding purpose. Because of its many technical advantages like accuracy, repeatability (Position repeatability of $0.05 \mathrm{~mm}$ and Path repeatability of $0.35 \mathrm{~mm}$ ) and its drivability. The weight of the robot is $170 \mathrm{Kg}$. Also, the emission from the robot is EMI Shielded. This is generally used in the industry and research work. Therefore, this model is selected for the forward and inverse kinematic analysis.

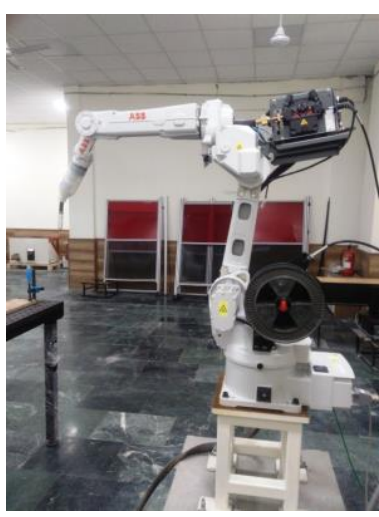

Fig.1 (a)

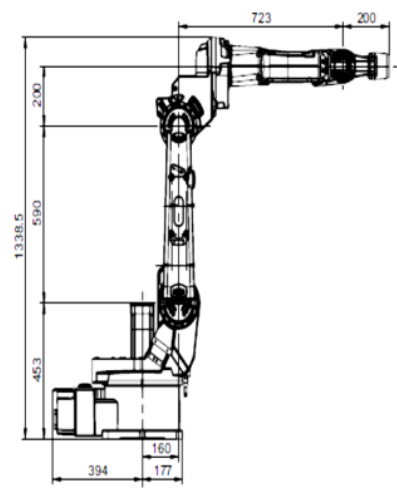

Fig.1 (b)
Fig. 1: (a) ABB IRB 1520 Industrial Robot, Robotics Laboratory, G L Bajaj Institute of technology \& management , Greater Noida (b) Manipulator dimensions ${ }^{14)}$

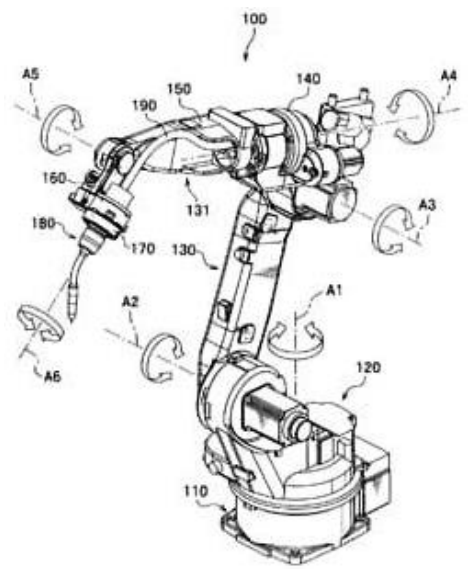

Fig. 2: A three-dimensional 6 DOF ABB IRB 1520 Robot manipulator $^{14)}$ 
Figure 2 shows the configuration of a robot manipulator in three dimensions. The manipulator has total 6 joint axes and 6DOF: Joint 1 (type $T$ joint) permits rotation about Z-axis. Joints 2,3 (type $\mathrm{R}$ ) allow rotation about an axis perpendicular to Z-axis. Joint 4 (type $\mathrm{T}$ joint) permits rotation about $\mathrm{X}$-axis. Joint 5 allows rotation about Y-axis, and joint 6 allows rotation about $\mathrm{X}$-axis.

The coordinate of each axis are shown in the figure 2 where the rotation is allowed. Each joint has its minimum and a maximum rotation limit which is constrained by the mechanical stopper and the motion is sense by the sensors attached at each joint. The base axis is permitted to rotate about $340^{\circ}\left( \pm 170^{\circ}\right)$ about axis A1. Other joints have constraint motions about their rotational axes.

On the basis of the defined robot arrangement the kinematic analysis has done. It is divided into forward and inverse kinematic analysis which is presented in the subsequent sections.

\section{Forward Kinematics (FKin)}

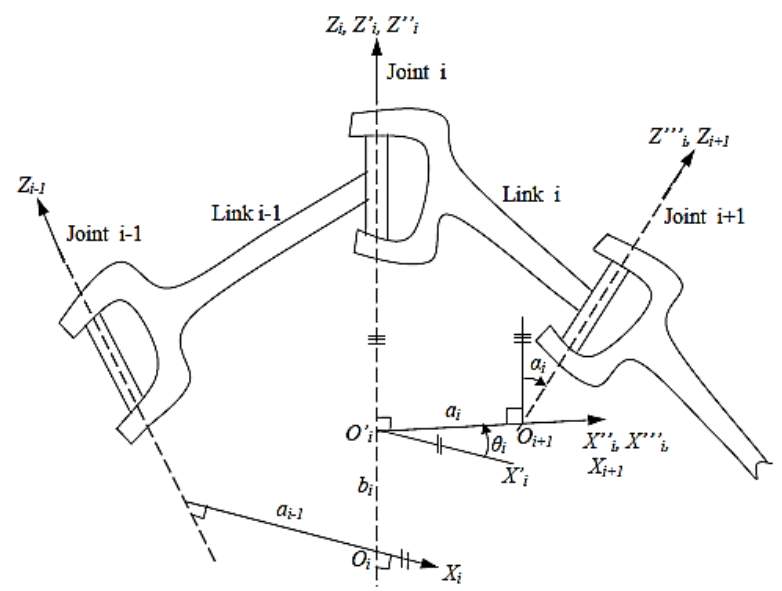

Fig. 3: Frame (i) description with respect to frame (i-1) ${ }^{12,13)}$

A robot manipulator has several links which are connected with a revolute or prismatic joint. Therefore every joint will have a single degree of freedom and in order to find the position of end effector (EE) with respect to its base it is important to find the relationship between the coordinate frames associated with the EE and the robot base. This can be done by applying the coordinate transformations between the coordinate frames to apply to each joint for kinematic analysis. For the calculation of $\mathrm{EE}$ position and orientation with respect to base or any axis, a mathematical model can be described by taking a product of TM of each manipulator joint. It is important to define a global coordinate system to the robot base and a joint coordinate system at each joint. D-H analysis is one of the major techniques used to evaluate the motion of the robot. It is used to write the TMs between the robot joint axes. These matrices involve four parameters having translation and rotations about the different axis.
The D-H analysis was presented in ${ }^{12)}$ to develop the TMs between the robot axes.

Table 1 Description of D-H parameters

\begin{tabular}{|c|c|}
\hline Parameters & Description \\
\hline bi (Joint offset) & $\begin{array}{l}\text { Distance between } \mathrm{Xi} \text { and } \mathrm{Xi}+1 \text { along } \\
\mathrm{Zi} \text { is bi }\end{array}$ \\
\hline$\theta \mathrm{i}$ (Joint angle) & $\begin{array}{l}\text { Angle between } \mathrm{Xi} \text { and } \mathrm{Xi}+1 \text { about } \mathrm{Zi} \\
\text { is } \theta \mathrm{i}\end{array}$ \\
\hline ai (Link length) & $\begin{array}{l}\text { Distance between the common normal } \\
\text { to axes } \mathrm{Zi} \text { and } \mathrm{Zi}+1 \text { along } \mathrm{Xi}+1 \text { is ai } \\
\text { (Link length) }\end{array}$ \\
\hline ai (Twist angle) & $\begin{array}{l}\text { Angle between the axes, } \mathrm{Zi} \text { and } \mathrm{Zi} \\
+1 \text {, about axis } \mathrm{Xi}+1 \text { (taken positive } \\
\text { when rotation is made counter } \\
\text { clockwise) }\end{array}$ \\
\hline
\end{tabular}

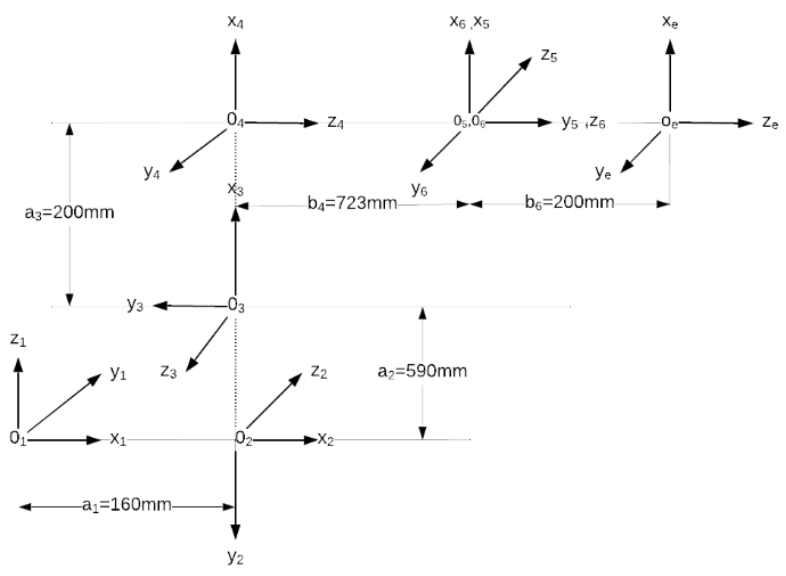

Fig. 4: ABB IRB 1520 frame assignments

These TMs involve four variables to establish relationships between succeeding links having translation and rotation axis. With reference to figure 4 the four D-H parameters are defined in the table 1 . It may be noted that these four parameters are explained in a sequence because these parameters are calculated from the link (i-1) to link (i+1) through link i. The relative linear and angular positions of the two links (i-1) and $i$ are defined in the joint offset (bi) and joint angle ( $\theta$ i). Whereas, size and shape of the link $\mathrm{i}$ are described in the last two parameters i.e. Link length (ai) and Twist angle ( $\alpha \mathrm{i})$ and they are remaining constant. Parameters bi and $\theta i$ are considered as variables which depend upon the kinematic arrangement. If joint $\mathrm{i}$ is prismatic then bi is taken as variable and if joint $i$ is revolute then $\theta i$ is taken as variable.

So according to the type of joint and their connectivity i.e. prismatic or revolute, one parameter is taken as variable which is known as joint variable (JV) and other parameters are constant, known as link parameters'. Next step is to assign the frames for the joint. 
Table 2 D-H parameters of ABB IRB 1520 Industrial Robot [JV: Joint variable]

\begin{tabular}{|c|c|c|c|c|}
\hline Links & $\begin{array}{c}\text { bi (Joint } \\
\text { offset, } \\
\text { mm) } \\
\end{array}$ & $\begin{array}{c}\theta \mathbf{i} \\
(\text { Joint } \\
\text { angle })\end{array}$ & $\begin{array}{c}\text { ai (Link } \\
\text { length, } \\
\text { mm) }\end{array}$ & $\begin{array}{c}\alpha i \\
\text { (Twist } \\
\text { angle) }\end{array}$ \\
\hline 1. & $b_{1}=453$ & $\theta_{1}(\mathrm{JV})$ & $a_{1}=160$ & $-90^{0}$ \\
\hline 2. & 0 & $\theta_{2}(\mathrm{JV})$ & $a_{2}=590$ & $180^{\circ}$ \\
\hline 3. & 0 & $\theta_{3}(\mathrm{JV})$ & $a_{3}=200$ & $90^{\circ}$ \\
\hline 4. & $b_{4}=723$ & $\theta_{4}(\mathrm{JV})$ & 0 & $90^{\circ}$ \\
\hline 5. & 0 & $\theta_{5}(\mathrm{JV})$ & 0 & $-90^{0}$ \\
\hline 6. & $b_{6}=200$ & $\theta_{6}(\mathrm{JV})$ & 0 & $0^{0}$ \\
\hline
\end{tabular}

In the figure 4, frames have been assigned for the different axis of rotations for the calculation of $\mathrm{D}-\mathrm{H}$ parameters. For the frame assigning of the manipulators axis first $\mathrm{Z}$ axis has to select then decide the direction of the $X$ axis according to the rotation of the joints. The forward kinematic analysis can be done with the help of the Eq 1. Where [Mi] and [Mee] represent the TM corresponding to $\mathrm{i}^{\text {th }}$ joint's D-H parameters as described in table 2, and that of the end effector with respect to base frame respectively,

$\left[\mathrm{M}_{\mathrm{ee}}\right]=$ Transformation Matrix of the end effector

$\left[\mathrm{M}_{\mathrm{ee}}\right]=\left[\mathrm{M}_{1}\right] *\left[\mathrm{M}_{2}\right] *\left[\mathrm{M}_{3}\right] *\left[\mathrm{M}_{4}\right] *\left[\mathrm{M}_{5}\right] *\left[\mathrm{M}_{6}\right]$

Where,

$$
\begin{aligned}
{\left[\mathrm{M}_{1}\right] } & =\left[\begin{array}{cccc}
\cos \theta_{1} & 0 & -\sin \theta_{1} & \mathrm{a}_{2} \cos \theta_{1} \\
\sin \theta_{1} & 0 & \cos \theta_{1} & \mathrm{a}_{2} \sin \theta_{1} \\
0 & -1 & 0 & \mathrm{a}_{1} \\
0 & 0 & 0 & 1
\end{array}\right] \\
{\left[\mathrm{M}_{2}\right] } & =\left[\begin{array}{cccc}
\sin \theta_{2} & \cos \theta_{2} & 0 & \mathrm{a}_{3} \cos \theta_{2} \\
\cos \theta_{2} & \sin \theta_{2} & 0 & \mathrm{a}_{3} \sin \theta_{2} \\
0 & 0 & -1 & \mathrm{a}_{2} \\
0 & 0 & 0 & 1
\end{array}\right] \\
{\left[\mathrm{M}_{3}\right] } & =\left[\begin{array}{ccccc}
\cos \theta_{3} & 0 & \sin \theta_{3} & \mathrm{a}_{4} \cos \theta_{3} \\
\sin \theta_{3} & 0 & \cos \theta_{3} & \mathrm{a}_{4} \sin \theta_{3} \\
0 & 1 & 0 & \mathrm{a}_{3} \\
0 & 0 & 0 & 1
\end{array}\right] \\
{\left[\mathrm{M}_{4}\right] } & =\left[\begin{array}{ccccc}
\cos \theta_{4} & 0 & \sin \theta_{4} & \mathrm{a}_{5} \cos \theta_{4} \\
\sin \theta_{4} & 0 & \cos \theta_{4} & \mathrm{a}_{5} \sin \theta_{4} \\
0 & 0 & 0 & \mathrm{a}_{4} \\
0 & 1 & 0 & 1
\end{array}\right] \\
{\left[\mathrm{M}_{5}\right] } & =\left[\begin{array}{ccccc}
\cos \theta_{5} & 0 & -\sin \theta_{5} & \mathrm{a}_{6} \cos \theta_{5} \\
\sin \theta_{5} & 0 & \cos \theta_{5} & \mathrm{a}_{6} \sin \theta_{5} \\
0 & -1 & 0 & \mathrm{a}_{5} \\
0 & 0 & 0 & 1 \\
0 & & 0 & \mathrm{a}_{7} \cos \theta_{6} \\
\cos \theta_{6} & -\sin \theta_{6} & 0 & \mathrm{a}_{6} \\
\sin \theta_{6} & \cos \theta_{6} & 0 & \mathrm{a}_{7} \sin \theta_{6} \\
0 & 0 & 1 & 1 \\
0 & 0 & 0 & 1
\end{array}\right]
\end{aligned}
$$

The transformation matrix $\left[\mathrm{M}_{\mathrm{ee}}\right]$ describes the position and orientation of $\mathrm{EE}$ with respect to robot base reference frame ${ }^{15,16)}$. These matrices are converted into equations which are further calculated with the help of MATLAB programming. End effector trajectories are plotted in the space and its maximum reach should be decided first in order to avoid the singularity criteria. Singularity of the robot manipulator is the situation where the robot motion is affected. This is the configuration of the robot manipulator where the position of the end effector is blocked. In some cases of robots the singularity can be easily identified but in some cases it is impossible to identify without using the complex mathematical complex equations. Therefore it is necessary to consider the singularity of the manipulators carefully before analysis the kinematics.

For the forward kinematic analysis it was required to select the coordinates of different positions of the end effectors in the space. A set of eight positions as described in the figure 5 were randomly chosen to obtain FKin model. Positions at wrist centre with axis of rotation are selected in order to describe $\mathrm{X}$ and $\mathrm{Z}$ position vectors from the table 3 . It may be noted that there is no $\mathrm{Y}$ component to these particular positions because $\theta_{1}$ is always selected as zero. These positions are used to entre in the software used for the analysis purpose and the same values were used in the MATLAB programming. Therefore the validity of the model has been confirmed with the help of programming and is presented in the subsequent results and discussion.

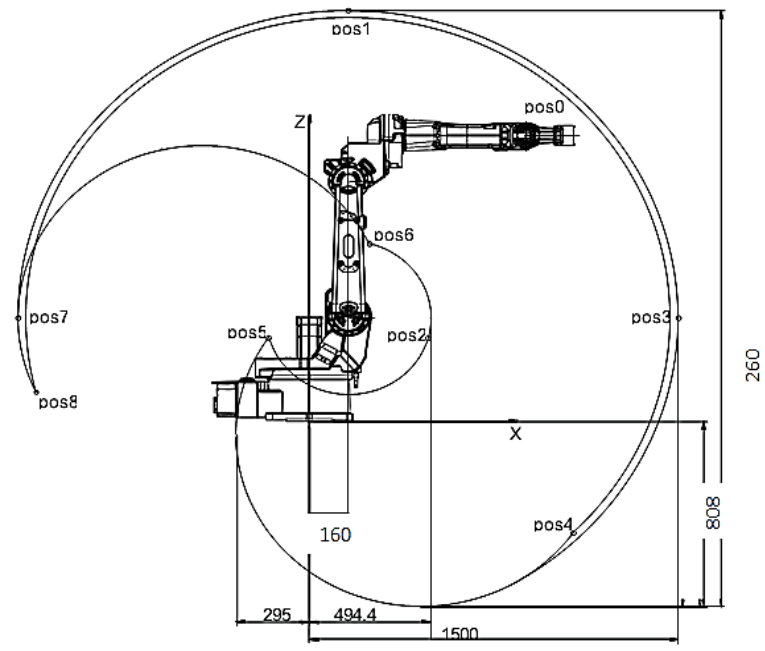

Fig.5: Positions at wrist center and working range with extra mechanical stop on axis $3^{14)}$

Table 3 Positions at wrist center with axis rotation

\begin{tabular}{|c|c|c|c|c|}
\hline Positions & $\begin{array}{c}\mathbf{X} \\
\text { Position } \\
(\mathbf{m m})\end{array}$ & $\begin{array}{c}\mathbf{Z} \\
\text { Position } \\
(\mathbf{m m})\end{array}$ & $\begin{array}{c}\text { Joint Axis } \\
\text { 2 Angle } \\
\text { (degrees) }\end{array}$ & $\begin{array}{c}\text { Joint Axis } \\
\text { 3 Angle } \\
\text { (degrees) }\end{array}$ \\
\hline P0 & 881 & 1242 & 0 & 0 \\
\hline P1 & 161 & 1792 & 0 & -73.4 \\
\hline P2 & 484 & 364 & 0 & +80 \\
\hline
\end{tabular}




\begin{tabular}{|c|c|c|c|c|}
\hline P3 & 1502 & 452 & +90 & -73.4 \\
\hline P4 & 1072 & -482 & +150 & -100 \\
\hline P5 & -162 & 366 & +150 & +81 \\
\hline P6 & 246 & 775 & -90 & +81 \\
\hline P7 & -1181 & 451 & -90 & -74.4 \\
\hline P8 & -1106 & 129 & -90 & -98.7 \\
\hline
\end{tabular}

All the joints have a minimum and maximum rotation limits because all of them cannot be fully rotated by $360^{\circ}$. These rotational limits of movement of different joints are presented in the table 4 . In this table the type of motion and their maximum and minimum range of movements are defined at which the robot manipulator can move. This table will be further used to IKin analysis. The detailed procedure of IKin analysis is presented in the subsequent sections.

Table 4 ABB IRB 1520 range of movement ${ }^{14}$

\begin{tabular}{|c|c|c|}
\hline Axis descriptions & Type of motion & $\begin{array}{c}\text { Range of } \\
\text { Movement }\end{array}$ \\
\hline A 1 & Rotation motion & $+170^{\circ}$ to $-170^{\circ}$ \\
\hline A 2 & Arm motion & $+150^{\circ}$ to $-90^{\circ}$ \\
\hline A 3 & Arm motion & $+80^{\circ}$ to $-100^{\circ}$ \\
\hline A 4 & Rotation motion & $+155^{\circ}$ to $-155^{\circ}$ \\
\hline A5 & Bend motion & $+135^{\circ}$ to $-135^{\circ}$ \\
\hline A 6 & Turn motion & $+200^{\circ}$ to $-200^{\circ}$ \\
\hline A 7 & Rotation motion & $+170^{\circ}$ to $-170^{\circ}$ \\
\hline
\end{tabular}

\section{Inverse kinematics}

As compared to the forward kinematics of the robot manipulator the inverse kinematic problem is difficult to solve. The inverse kinematics robotics problem focuses on kinematic analysis of robotics manipulator. There are some problems associated with the Ikin for example Non-linear equations, the multiple solutions existence, possibility of non-existence solutions and mechanism singularities. In order to determine solution all possible formations to move from end effector position to base position, one must compute the movements associated with each joint variable. In case of FKin the problem has only one solution as the parameters of the end effector are determined uniquely. However, in case of the IKin the problem has several solutions and these needs to be derived very carefully. There are both conventional and in conventional approach available to find the solution. Raghvan and Roth have suggested a method to find out the IKin for serial $6 \mathrm{R}$ manipulator robot ${ }^{17)}$. IKin analysis of a generic $6 \mathrm{R}$ serial manipulator with the integration of RoboAnalyzer software was performed ${ }^{18,19)}$.

To obtain IKin of ABB IRB 1520 robot the equation 1 is rewritten as

$$
\text { Mee }=\mathrm{M}_{1} *[\text { Mee }]_{2} \text {; }
$$

Where, $[\mathrm{Mee}]_{2}=\left[\mathrm{M}_{2}\right] *\left[\mathrm{M}_{3}\right] *\left[\mathrm{M}_{4}\right] *\left[\mathrm{M}_{5}\right] *\left[\mathrm{M}_{6}\right]$

In the above Eq $2\left[\mathrm{Mee}_{2}\right.$ corresponds to the TM of the EE with respect to the frame 2 as shown in figure 5. It may be noted that the EE point always lies in the $\mathrm{XY}$ plane of frame 2 when joint 1 rotates, and therefore this expression does not include $\theta_{1}$. This is just because of the robot architecture. Therefore, the calculation of angles of rotation of joint 2 , joint 3 , joint 4 and joint 5 is simply reduced to a planar problem. Thus $[\mathrm{Mee}]_{2}$ can be determined as

$[\mathrm{Mee}]_{2}=\mathrm{M}_{1}^{-1} *$ Mee

Where, $\mathrm{M}_{1}^{-1}$ corresponds to the TM of frame of joint 1 with respect to that of joint 2 . Note that $M_{1}$ was calculated in the FKin by simply multiplying four individual TM referring to each of the D-H parameters for joint 1 . In the same way $\mathrm{M}_{1}^{-1}$ can be calculated by multiplying TMs referring to the opposite sign value of each of the D-H parameters and the order of multiplication is kept opposite. The expression for $\mathrm{M}_{1}^{-1}$ is obtained for $\alpha_{1}=90^{\circ}$ as

$$
\mathrm{M}_{1}^{-1}=\frac{\operatorname{Adj}\left(\mathrm{M}_{1}\right)}{\left|\mathrm{M}_{1}\right|}
$$

Where;

$\operatorname{Adj}\left(M_{1}\right)=$ Transpose of cofactor of matrix $\left(M_{1}\right)$, and

$$
\left|M_{1}\right|=\text { Determinant of }\left(M_{1}\right)
$$

$$
\left[\mathrm{M}_{1}^{-1}\right]=
$$$$
\left[\begin{array}{cccc}
\cos \theta_{1} & \sin \theta_{1} & 0 & \left(-\sin \theta_{1} \mathrm{a}_{2} \sin \theta_{1}-\cos \theta_{1} \mathrm{a}_{2} \cos \theta_{1}\right) \\
0 & 0 & -1 & \mathrm{a}_{1} \\
-\sin \theta_{1} & \cos \theta_{1} & 0 & \left(\tan \theta_{1} \mathrm{a}_{2} \cos \theta_{1}-\mathrm{a}_{2} \sin \theta_{1}\right) \mathrm{a}_{2} \cos \theta_{1} \\
0 & 0 & 0 & 1
\end{array}\right]
$$

In equation 4 the TM does not have a $\mathrm{Z}$ component in the last column. This indicates clearly that end effector point will always remain on the XY plane of frame 2 .

Let $\mathrm{V}=\left[\mathrm{V}_{\mathrm{x}}, \mathrm{V}_{\mathrm{y}}, \mathrm{V}_{\mathrm{z}}\right] \mathrm{T}$ refer to the position of EE with respect to frame 1 , and

$$
\mathrm{V}^{\prime}=\left[\mathrm{V}_{\mathrm{x}}{ }^{\prime}, \mathrm{V}_{\mathrm{y}}{ }^{\prime}, \mathrm{V}_{\mathrm{z}}{ }^{\prime}\right] \mathrm{T} \text {. }
$$

The IKin of ABB IRB 1520 robot arm can be simplified in the following manner:

Angle $\theta_{1}$ has only contributed to $\mathrm{V}_{\mathrm{x}}$ and $\mathrm{V}_{\mathrm{y}}$ and does not affect $\mathrm{V}_{\mathrm{z}}$. With the help of the geometry, $\theta_{1}$ can be determined as:

$$
\theta_{1}=\mathrm{a} \tan ^{2}\left(\mathrm{~V}_{\mathrm{x}}, \mathrm{V}_{\mathrm{z}}\right)
$$

Origins of coordinate system of frame 2 to frame 6 always lie on XY plane of frame 2. And this plane rotates with respect to YZ plane of frame 1. Therefore, finding kinematic parameters of end effector point in frame 2 can be reduced to a planar problem. Also, the rotation of last joint 6 does not affect the position of the EE in frame 2 . Therefore, the expression for the EE point in frame 2. (V') is given by:

$$
\left[\mathrm{V}^{\prime}\right]=\left[\mathrm{M}_{1}^{-1}\right] *[\mathrm{~V}]
$$




$$
\left[\mathrm{V}^{\prime}\right]=\left[\begin{array}{c}
\mathrm{Vx}^{\prime} \\
\mathrm{Vy}^{\prime} \\
\mathrm{Vz}^{\prime} \\
0
\end{array}\right]=\left[\begin{array}{cccc}
\cos \theta_{1} & \sin \theta_{1} & 0 & -\mathrm{a}_{2} \\
0 & 0 & -1 & \mathrm{a}_{1} \\
-\sin \theta_{1} & \cos \theta_{1} & 0 & 0 \\
0 & 0 & 0 & 1
\end{array}\right]\left[\begin{array}{c}
\mathrm{Vx} \\
\mathrm{Vy} \\
\mathrm{Vz} \\
0
\end{array}\right]
$$

(c) Once [V'] is determined from the actual input for IKin [V], the calculations for joint angles $\theta_{2}, \theta_{3}, \theta_{4}$ and $\theta_{5}$ can be done similar to a planar $3 \mathrm{R}$ serial robot as derived in $^{11)}$. The results of IKin were obtained by using MATLAB program and compared with the results obtained for the same $\mathrm{DH}$ parameters with help of RoboAnalyzer software.

\section{Results and Discussions}

The forward kinematics (FKin) and inverse kinematics (IKin) of the robot is carried out using RoboAnalyzer software. The results of forward kinematics computed using MATLAB with proposed mathematical model is compared with those of RoboAnalyzer software. The plots of the end-effector coordinate produced using RoboAnalyzer is given in Fig. 7 and those of MATLAB in Fig. 8 respectively. The results are perfectly matched, hence, validate the proposed model. The results of predicts that the initially the y-coordinate of end-effector is zero. This depends on the choice of initial joint coordinates are taken.

The cycloidal joint trajectories considered for the forward kinematics is given as ${ }^{11)}$ :

$$
\theta_{i}=\theta_{i}(0)+\frac{\theta_{i}(T)-\theta_{i}(0)}{T}\left\lfloor t-\frac{T}{2 \pi} \sin \left(\frac{2 \pi}{T} t\right)\right\rfloor \quad \text { for } i=1,2,3,4,5,6
$$

Where, $\theta_{\mathrm{i}}(0)$ is the initial configuration, $\theta_{\mathrm{i}}(\mathrm{T})$ is the final configuration, $\mathrm{T}=$ time taken i.e. $5 \mathrm{sec}, \mathrm{t}=$ instant time, sec

Major advantage of selection of cycloidal trajectory is that when robot start and end with zero acceleration it will not experience any jerk or vibration. Due to which the robot motion is very smooth. Therefore, if the travel time is selected relatively large then this trajectory is most suitable. At the same time the total energy consumption as compare to other trajectories is also less.

Selected robot generated in the FKin and IKin can be easily analyzed without using any typical programming in MAT lab ${ }^{20,21,22,23)}$. The FKin and IKin of ABB IRB 1520 robot was calculated with the integration of RoboAnalyzer. The joint trajectories of the robot links are generated in the form of graph after defining the D-H parameters. The simulation of the robot links results can be visualized in the form of graph. It was assumed that there are no linkages joint deformation and satisfy all structural strength criteria ${ }^{24,25,26,27)}$.

\subsection{Forward Kinematics Analysis of ABB IRB 1520 Robot}

In FKin analysis of the ABB IRB 1520 Robot by using software first the D-H parameter obtain earlier section are entered in the software to get the 3D model of the robot as shown in the figure 6 . In this model frames are assigned to all the axis of rotations. For the FKin analysis the total time taken and numbers of steps are selected and run the program. Once the FKin analysis is completed then the trajectories of the EE can be easily obtained.

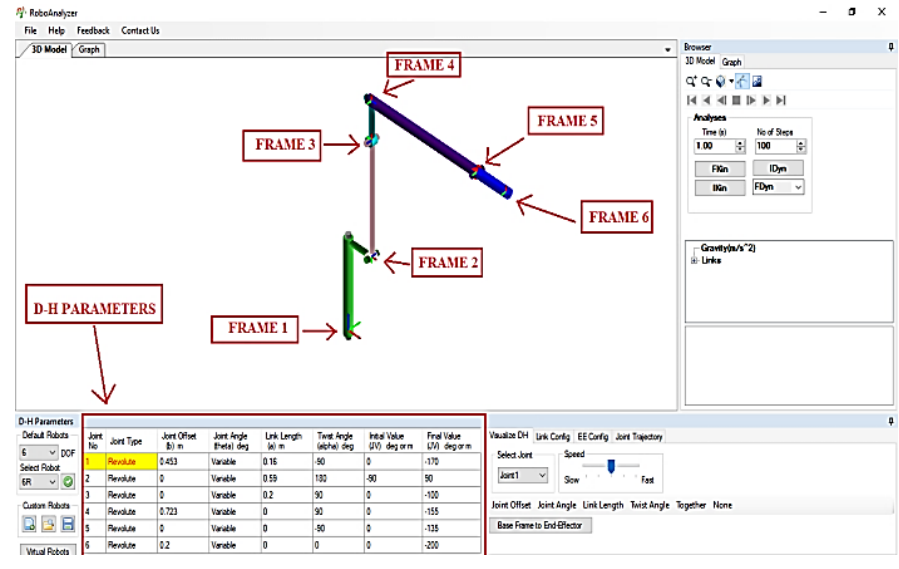

Fig. 6: 3D view of ABB IRB 1520 with DH parameters of robot in RoboAnalyzer

Since this model is not available in the software, a CAD model was used whose shape did not match exactly with the real robot. Since links length and center to center distances between the joints are based on the D-H parameters and are defined in earlier section in the table 3 , therefore this did not affect the kinematic analysis of the robot. After developing the CAD model in the software, the program is run for predefined number of steps and work cycle to simulate the robot and get the trajectory of the $\mathrm{EE}$ as presented in figure 7.

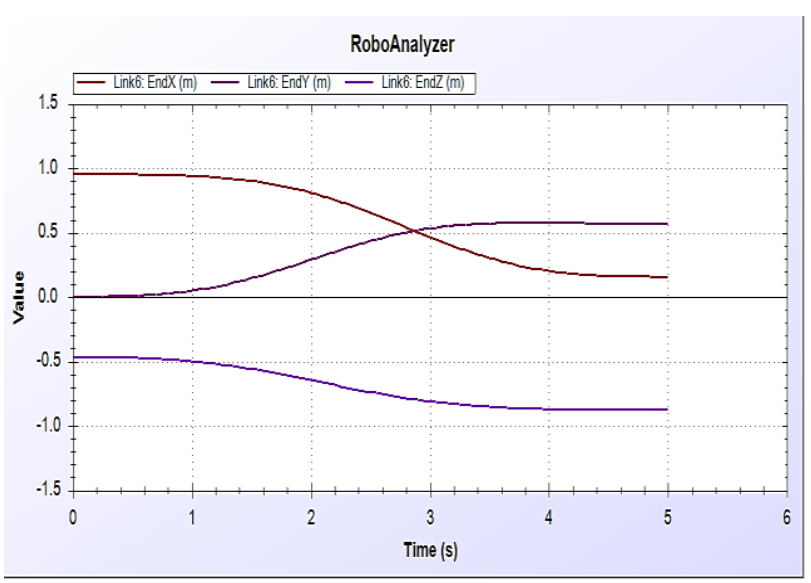

Fig. 7: Trajectory of end effector of ABB IRB 1520 robot obtained in RoboAnalyzer

The initial position of the EE in $\mathrm{X}, \mathrm{Y}$ and $\mathrm{Z}$ coordinates are selected as $1.0,0$, and -0.5 with the consideration of cycloidal curve and the final position of the EE after $5 \mathrm{sec}$ were computed. This has been noted that the same values and trajectories were computed with the help of MAT lab 
programing. This comparison validates the mathematical formulation of the FKin analysis. Other link trajectories with respect to time in $5 \mathrm{sec}$ of the various joints of the manipulator are also analysed and shown in the figure 9.

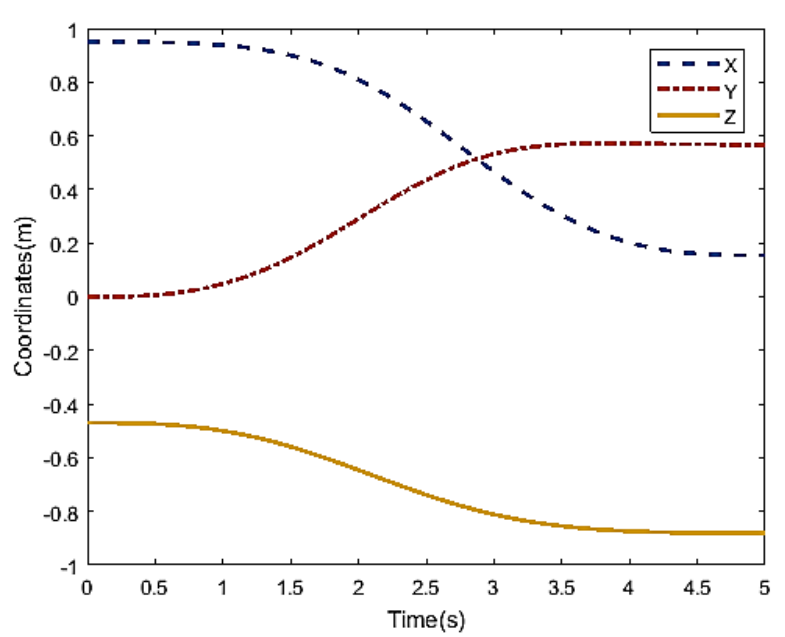

Fig. 8: End effector trajectory of ABB IRB obtained with MATLAB Program

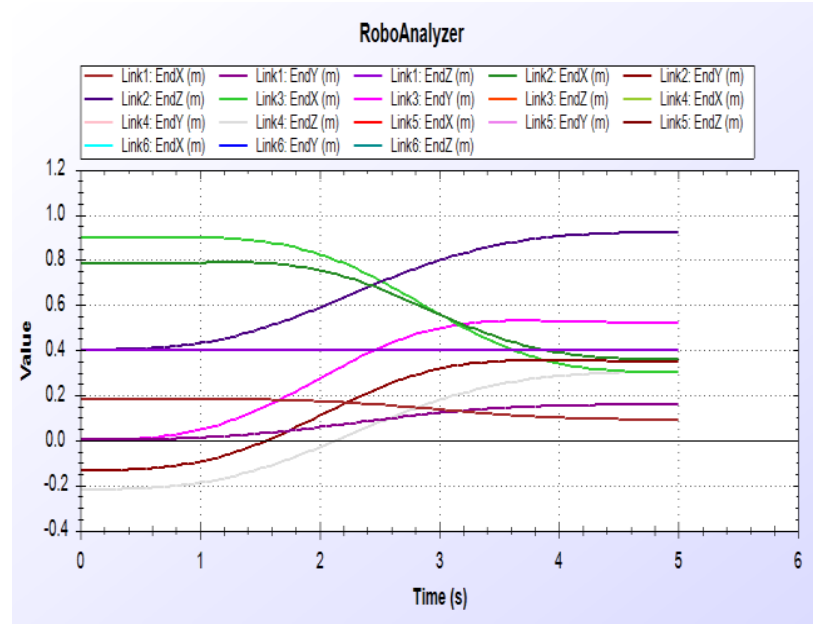

Fig. 9: Links trajectories with respect to time in 5 sec.

\subsection{Homogeneous matrix calculation with respect to base frame:}

This section presents the transformation matrix between the frame attached to the links.

$$
\begin{aligned}
& {\left[\mathrm{M}_{1}\right]=\left[\begin{array}{cccc}
1 & 0 & 0 & 0.16 \\
0 & 0 & 1 & 0 \\
0 & -1 & 0 & 0.45 \\
0 & 0 & 0 & 1
\end{array}\right]} \\
& {\left[\mathrm{M}_{2}\right]=\left[\begin{array}{cccc}
1 & 0 & 0 & 0.75 \\
0 & 0 & -1 & 0 \\
0 & 1 & 0 & 0.45 \\
0 & 0 & 0 & 1
\end{array}\right],}
\end{aligned}
$$

$$
\begin{aligned}
& {\left[\mathrm{M}_{3}\right]=\left[\begin{array}{cccc}
1 & 0 & 0 & 0.95 \\
0 & -1 & 0 & 0 \\
0 & 0 & -1 & 0.45 \\
0 & 0 & 0 & 1
\end{array}\right]} \\
& {\left[\mathrm{M}_{4}\right]=\left[\begin{array}{cccc}
1 & 0 & 0 & 0.95 \\
0 & 0 & 1 & 0 \\
0 & -1 & 0 & -0.27 \\
0 & 0 & 0 & 1
\end{array}\right]} \\
& {\left[\mathrm{M}_{5}\right]=\left[\begin{array}{cccc}
1 & 0 & 0 & 0.95 \\
0 & -1 & 0 & 0 \\
0 & -0 & -1 & -0.27 \\
0 & 0 & 0 & 1
\end{array}\right]} \\
& {\left[\mathrm{M}_{6}\right]=\left[\begin{array}{cccc}
1 & 0 & 0 & 0.95 \\
0 & -1 & 0 & 0 \\
0 & 0 & -1 & -0.47 \\
0 & 0 & 0 & 1
\end{array}\right]} \\
& {\left[\mathrm{M}_{\mathrm{ee}}\right]=\left[\begin{array}{cccc}
1 & 0 & 0 & 0.95 \\
0 & -1 & 0 & 0 \\
0 & 0 & -1 & -0.47 \\
0 & 0 & 0 & 1
\end{array}\right]}
\end{aligned}
$$

\subsection{Inverse kinematics of ABB IRB 1520 robot}

The inverse kinematics of the ABB IRB 1520 robot is performed only with RoboAnalyzer. The inverse kinematics of the serial-chain robots are quite challenging task. For each end-effector position there could be eight solutions corresponding to joint coordinates. Fig.10. shows the results of inverse kinematics with RoboAnalyzer software.

After getting the results of forward kinematics, the inverse kinematics of the ABB IRB 1520 robot can be obtained from RoboAnalyzer. Since in case of inverse kinematics there are multiple solutions which provide extra degree of flexibility of the robot. Assume that if the robot is working in a crowded environment and if there are multiple configurations obtained for the same end effector position then the robot has to select most appropriate configuration to avoid any type of accident. There are mainly eight possible solutions of the inverse kinematics. Out of these solutions we can chose any two, between which the forward kinematics can be simulated. Every joint must satisfy the joint angle range limit for each solution. In order to get multiple solutions we feed end effector value $(\mathrm{x}=0.1 \mathrm{~m}, \mathrm{y}=0.1 \mathrm{~m}$, and $\mathrm{z}=0.1 \mathrm{~m})$. Total eight solutions were obtained with the help of software used and it was found that two solutions were feasible out of eight depending upon the working conditions. These solutions are presented in the fig. 10 where the EE position was defined and eight possible manipulator configurations were obtained. 


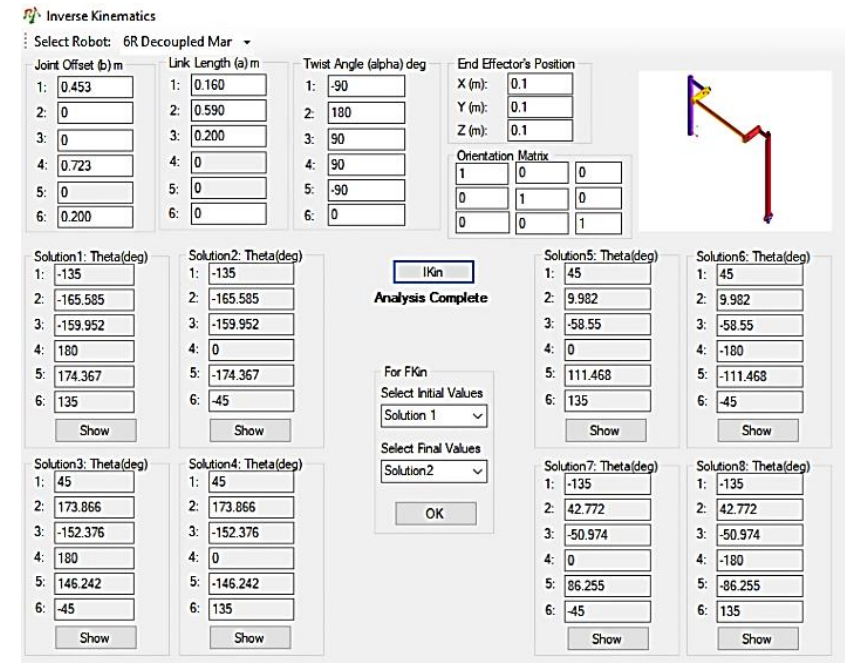

Fig. 10: Inverse kinematics of ABB IRB 1520 robot

\section{Nomenclature}

$\begin{array}{ll}\text { D-H } & \text { Denavit-Hartenberg } \\ \text { FKin } & \text { Forward Kinematics } \\ \text { IKin } & \text { Inverse Kinematics } \\ \text { IRB } & \text { Industrial Robot } \\ \text { (EE) } & \text { End effector } \\ \text { (TM)/M } & \text { homogeneous transformation matrix } \\ \text { DOF } & \text { Degrees of freedom } \\ \text { EMI } & \text { Electromagnetic interference } \\ \text { TM } & \text { Transformation Matrix } \\ \text { JV } & \text { Joint variable } \\ \text { V } & \text { Position Vector } \\ \text { R } & \text { Revolute Joint } \\ \text { T } & \text { Twisting Joint }\end{array}$

\section{Conclusions}

In this study the forward and inverse kinematics is performed for the industrial robot ABB IRB 1520. A methodology is developed in the present work for kinematic analysis of the linkage and RoboAnalyzer software was used to simulate the motion. ABB IRB 1520 robot was selected for the forward and reverse kinematic analysis for which first D-H parameters were defined and the same parameters were used to simulate its motion in the software. The forward kinematics is calculated by moving the joints with cycloidal trajectory. On this trajectory the total time taken as compared to other trajectories are less. Also the motion of robot in the cycloidal trajectory is smooth and vibration free. The results of codes written in MATLAB for forward dynamics of any 6-DOF serial chain robot with revolute joints are compared with that of RoboAnalyzer software and found same presents the correctness of the approach used. Also, the inverse kinematics is also performed using inverse kinematics module of RoboAnalyzer for $6 \mathrm{R}$ robot. The complete forward and inverse kinematic equations are not presented in this paper due to the space restrictions. A MATLAB programming was used to solve the equations and results were obtained. These results were validated with the help of RoboAnalyzer software. These two results were found to be nearly same and therefore the kinematic analysis formulation was validated. Next we will try to validate the results with actual robot motion.

\section{Acknowledgement}

The authors are grateful to Prof S K Saha, Department of Mechanical Engineering, IIT Delhi for his valuable support and providing technical assistance through a technical workshop on RoboAnalyzer software was conducted by Dr. Paramanand V. Nandihal, Post-Doctoral Researcher, Mechatronics lab Mechanical Engineering Department, IIT, Delhi.

\section{References}

1) A.F. Nicolescu, Industrial Robots, EDP Publishing House, Bucharest, 2005.

2) G. Khushdeep, D. Sethi, An analytical method to find workspace of a robotic manipulator, Journal of Mechanical Engineering, Vol. 41, No. 1, 2010, pp. 25-30.

3) T. Yong, F. Chen, X. Hegen, Kinematics and Workspace of a 4-DOF Hybrid Palletizing Robot, Advances in Mechanical Engineering, Vol. 20, 2014.

4) A. Srikanth, M. Sravanth, V. Sreechand, K.K. Kumar, Kinematic Analysis of 3 dof of serial robot for industrial applications, International Journal of Engineering Trends and Technology (IJETT), Vol. 4, Iss. 4, 2013, pp.1000-1004.

5) K.K. Kumar, A. Srinath, G. Jugalanvesh, P. Premsai, M. Suresh, Kinematic Analysis and Simulation of a 6 DOF Kuka KR5 Robot for Welding Application, International Journalof Engineering Research and Applications (IJERA), Vol. 3, Issue 2, 2013, pp. 820-827.

6) T. Yong, F. Chen, X. Hegen, Kinematics and Workspace of a 4-DOF Hybrid Palletizing Robot, Advances in Mechanical Engineering, Vol. 2014, 2014.

7) Nicolescu, Adrian \& Ilie, Florentin-Marian \& Tudor - George, Alexandru., Forward and inverse kinematics study of industrial robots taking into account constructive and functional parameter's modeling. Proceedings in Manufacturing Systems. 10. 157. (2015)

8) Y. Xu, M.C. Nechyba, Fuzzy inverse kinematic mapping:Rule generation, efficiency, and implementation, Proceedings of the 1993 IEEE/RSJ International Conference, Vol. 2, 1993, pp. 911-918.

9) M. Dahari and J. Tan. Forward and inverse kinematics model for robotic welding process using kr-16ks kuka robot. 2011 Fourth International 
Conference on Modeling, Simulation and Applied Optimization, 2011.

10) R. N. Jazar, Theory of applied robotics vol. 1: Springer, 2010.

11) S. K. Saha, Introduction to Robotics, Second Edition, Tata McGraw Hill, New Delhi, 2014.

12) Mikell P Groover Industrial Robotics, Second Edition, McGraw Hill,2012

13) D. Pieper, The Kinematics of Manipulators Under Computer Control, Ph.D. Thesis, Stanford University, 1968.

14) ABB Robotics, Product specification manual IRB 1520, from http://www.abb.com/Document ID: 3HAC043437-001,2012-2019.

15) J. J. Craig, Introduction to robotics: mechanics and control: Pearson/Prentice Hall Upper Saddle River, NJ, USA: 2005.

16) R.P Paul, Robot Manipulators: Mathematics, Programming and control, MIT Press, Cambridge, MA, 1981

17) M. Raghavan, and B. Roth, "Inverse Kinematics of the General 6R Manipulator and Related Linkages," ASME Journal of Mechanical Design, vol. 115, no. 3, pp. 502-508, 1993.

18) Sasanka Sekhar Sinha, Rajeev lochana G. Chittawadigi and Subir Kumar Saha "Inverse Kinematics for General 6R "Manipulators in RoboAnalyzer" The $5^{\text {th }}$ Joint International Conference on Multibody System Dynamics" June 24-28, 2018, Lisbon, Portugal

19) Amogh Patwardhan, Aditya Prakash, Rajeevlochana G. Chittawadigi "Kinematic Analysis and Development of Simulation Software for Nex Dexter Robotic Manipulator" Procedia Computer Science 133 (2018) 660-667

20) Rajeev lochana, S. K. Saha, 2011. RoboAnalyzer: 3D model based robotic learning software. In Proceedings of the International Conference on Multi Body Dynamics (Vijayawada, India, Feb 12-14, 2011), 3-13.

21) Rajeev lochana, C. G., Jain, A., Shah, S. V. and Saha, S. K. 2011. Recursive Robot Dynamics in RoboAnalyzer. In Proceedings of the 15th National Conference on Machines and Mechanisms (Chennai, India, Nov. 30-Dec. 2, 2011), 482-490.

22) Othayoth, Ratan S., Chittawadigi, Rajeev lochana G., Joshi, Ravi P., and Saha, Subir K. (2017) "Robot kinematics made easy using RoboAnalyzer software." Computer Applications in Engineering Education 25(5): 669-680.

23) Peter Corke. Robotics toolbox for matlab. pdf, 2014.

24) SS Chauhan, S C Bhaduri "Structural analysis of a Four-bar linkage mechanism of Prosthetic knee joint using Finite Element Method" EVERGREEN Joint Journal of Novel Carbon Resource Sciences \& Green Asia Strategy, Vol. 07, Issue 02, pp209-215, June, 2020

25) Amr Mohamed Metwally Ismaiel, "Fatigue Analysis of an Optimized HAWT Composite Blade" EVERGREEN Joint Journal of Novel Carbon Resource Sciences \& Green Asia Strategy, Vol. 04, Issue 02/03, pp. 1-6, September 2017

26) Nagendra Kumar Maurya, Vikas Rastogi, Pushpendra Singh, Experimental and Computational Investigation on Mechanical Properties of Reinforced Additive Manufactured Component, EVERGREEN Joint Journal of Novel Carbon Resource Sciences \& Green Asia Strategy, Vol. 06, Issue 03, pp207-214, September, 2019.

27) Shashi Prakash Dwivedi, Manish Maurya, Nagendra Kumar Maurya, Ashish Kumar Srivastava, Satpa Sharma, Ambuj Saxena, Utilization of Groundnut Shell as Reinforcement in Development of Aluminum Based Composite to Reduce Environment Pollution: a review. EVERGREEN Joint Journal of Novel Carbon Resource Sciences \& Green Asia Strategy. 07 ( 01): 15-25(2020) 